\title{
Review of: "The nucleotide messenger (p)ppGpp is an anti-inducer of the purine synthesis transcription regulator PurR in Bacillus"
}

\author{
Christophe Herman ${ }^{1}$, Alice Wen ${ }^{1}$ \\ 1 Baylor College of Medicine
}

Potential competing interests: The author(s) declared that no potential competing interests exist.

This is an outstanding article which investigates the first-known direct interaction of the nucleotide messenger and alarmone guanosine pentaphosphate, ( $\mathrm{p}$ )ppGpp, with a transcription factor in Bacillus. Specifically, (p)ppGpp was found to be able to bind the PurR repressor's effector binding domain to allosterically compete with PurR's previously known inducer, phosphoribosyl pyrophosphate (PRPP). A thorough set of experiments were performed to dissect the overlapping binding domains of (p)ppGpp and PRPP, the effect of ( $p) p p G p p$ competition with PRPP on PurR-DNA interaction, the promoter occupancy at PurR binding sites across the $B$. subtilis genome following (p)ppGpp induction, and the anti-induction of purine biosynthesis genes by (p)ppGpp through PurR repressor activity during entry of cells into stationary phase. Altogether, the findings show that ( $\mathrm{p}$ )ppGpp can directly regulate nucleotide synthesis in Bacillus by binding to a classical transcription repressor. While the concept of (p)ppGpp-induced inhibition of purine biosynthesis is established, the discovery that (p)ppGpp can directly modulate repressor activity introduces a new mechanism whereby (p)ppGpp relays stress-response signaling in Bacillus and highlights the versatility of ( $p$ )ppGpp as a second messenger. This work will allow the pursuit of novel experimental designs for future investigations of ( $p) p p G p p$ interactions both with PurR as well as with other protein candidates.

Major strengths of this study include the thorough structural and physiological profiling of an interactor of (p)ppGpp, PurR, identified from a forward screen of the entire Bacillus proteome. Experiments methodically dissected the nature of the interaction of PurR with ( $p$ )ppGpp and the functional consequences of this interaction on PurR repressor activity in the context of nutrient stress adaptation.

1. Differential radial capillary action of ligand assay (DRACALA) ${ }^{1}$, a tried and true method to detect interactors of low-molecular-weight ligands, was used to detect (p)ppGpp interactors in a proteomewide screen. Non-radiolabeled GTP was added as a competitor to enrich for ppGpp-specific interactors. DRaCALA is generally highly specific. False positives may occur due to spotting errors but can easily be identified visually and removed from analysis ${ }^{2}$. Validation of hits using purified protein further corrects for false positives ${ }^{3}$. In this study, PurR binding signal was enriched after GTP competitor addition, and 
the binding activity to both pppGpp and ppGpp was validated with purified untagged PurR. Furthermore, the use of two PurR homologs increased the confidence of the hit as screening was performed in $B$. anthracis while validation occurred in B. subtilis.

2. X-ray crystallography was performed to verify the ppGpp binding domain of PurR. Reassuringly, the binding pocket was found to be highly conserved among Firmicutes, suggesting a vital role of ppGppPurR interactions in the evolution of the phylum. Furthermore, specific residues important for the interaction with ppGpp or PRPP were identified and functionally validated using DRaCALA binding curves. Finally, the binding pocket of ppGpp was found to overlap with the binding pocket of PRPP, lending credibility to the hypothesis of allosteric competition between the two signaling molecules.

3. Electromobility shift assays and DNAse I footprinting were performed to lend further support to the existence of allosteric competition between ppGpp and PRPP for PurR binding.

4. CHIP enrichment of PurR binding sites before vs after nucleobase-induced repression of PurR operons were similar to CHIP enrichment before vs after ( $p$ )ppGpp induction, providing in vivo evidence of (p)ppGpp regulation of PurR repression. Controls using ( $p) p p G p p^{0}$ mutants were performed to exclude artifacts of induction. Reassuringly, PurR binding motifs were identified in most of these sites of PurR binding enrichment.

5. The authors were able to successfully translate in vitro structural analysis to an in vivo demonstration of the impact of PurR regulation by PRPP and ( $\mathrm{p}$ )ppGpp during amino acid abundance and starvation. Introduction of a mutation at a PRPP-interacting residue of PurR identified on crystallography raised the doubling time of the culture in amino acid replete medium by almost two-fold compared to the WT strain. Introduction of a mutation at (p)ppGpp-interacting residues of PurR identified on crystallography increased the lag time of culture outgrowth by one hour when the cells experienced an intentional amino acid downshift. These results validate the importance of PRPP and ( $p$ )ppGpp regulation of PurR activity during nutrient shifts.

Minor caveats of this study include the limited sensitivity of the screening method, the ambiguity between the impact of pppGpp vs ppGpp, suggestive CHIP enrichment results that require further PurR structural analysis to validate, and in vivo results suggesting possible limitations in structural findings and/or upregulation of PurR repression by non-(p)ppGpp means. These caveats are largely overshadowed by the strengths mentioned above, but they provide a stepping stone for further future refinement of these techniques.

1. While the DRaCALA binding assay is generally highly specific, it has a limited sensitivity due to variability in overexpression across the proteome ${ }^{3}$ and weak detection of low equilibrium ligand binders ${ }^{4}$. Therefore, a proteome-wide screen may not detect many many potential interactors. In addition, for this study, PurR binding signal was below the Z-score cutoff in the initial screen and was only enhanced above the cutoff upon the addition of GTP competitor, which may imply substantial 
variability and/or a higher baseline of non-specific ppGpp and GTP interactions in a majority of analyzed ORFs that could potentially be due to non-endogenous protein tags. However, the validation of pppGpp and ppGpp binding with purified untagged PurR protein at a physiologically relevant concentration of pppGpp and ppGpp is suggestive of meaningful interactions between the alarmone and PurR in vivo.

2. There is some difficulty in the study in distinguishing between PurR interactions with pppGpp vs interactions with ppGpp. While binding curves performed with purified PurR protein showed very similar $K_{d}$ between both pppGpp and ppGpp, it is still unclear whether there may be differences between the affinity of PurR for pppGpp vs ppGpp in vivo. Crystallography, EMSA, and DNAse I footprinting were performed with ppGpp, while CHIP-seq was performed with the general induction of ( $p$ )ppGpp by arginine hydroxamate. In the future, it may be interesting to quantify whether different methods of (p)ppGpp induction induce varied ratios of pppGpp vs ppGpp in Bacillus, and whether this has any impact on the role of (p)ppGpp-mediated regulation of binding partners. Differential strength of regulation has already been shown for $E$. coli, where ppGpp was found to be a more potent regulator than pppGpp of growth rate and RpoS induction ${ }^{5}$, and has been suggested in Bacillus, for which pppGpp seems to be a more potent inhibitor of DNA primase than ppGpp ${ }^{6}$.

3. In many cases, CHIP binding near PurBox sites showed peak enrichment off-center from the PurBoxes themselves, and the authors hypothesize that PurR may nucleate outwards from the initial binding site. Structural data also shows that upon ppGpp binding, the structure of a loop in PurR that bridges the monomer-monomer interface of PurR dimers stabilizes, revealing a positively charged channel. This is suggestive of a possible alteration of PurR affinity for the electronegative DNA backbone, potentially leading to DNA wrapping around the dimer and/or nucleation of multiple PurR dimers. However, structural data of ppGpp-bound PurR in conjunction with PurBox sequences may be necessary to resolve whether nucleation vs DNA wrapping occurs. Furthermore, as the direction and distance of peak enrichment in relation to the PurBoxes varies with each binding site on the genome, closer analysis of the PurBox-adjacent sequences at each genomic site may provide more clarity on whether there is a sequence-specific direction of nucleation or DNA wrapping.

4. In vivo data demonstrates only a one-hour increase in lag time of (p)ppGpp-unresponsive PurR mutants compared to the WT strain following amino acid downshift. This delay of outgrowth by only one hour could suggest either 1 ) the structural mutations in PurR that render it unresponsive to (p)ppGpp binding in vitro can be somehow overcome in vivo at higher ( $p$ )ppGpp concentrations, or 2 ) there are other non(p)ppGpp upregulators of PurR repression in vivo. Both possibilities are intriguing and would require further follow-up.

In conclusion, this work is a highly rigorous study with interesting findings that will advance our understanding of the bacterial starvation response. In particular, this study provides the first known documentation of alarmone (p)ppGpp's regulation of gene expression through direct interactions with transcription factors in Bacillus. The overarching story is well-supported by data from the multidisciplinary 
approaches taken.

1. Roelofs KG, Wang J, Sintim HO, Lee VT. Differential radial capillary action of ligand assay for highthroughput detection of protein-metabolite interactions. Proc Natl Acad Sci U S A. Sep 13 2011;108(37):15528-33. doi:10.1073/pnas.1018949108

2. Seminara AB, Turdiev A, Turdiev H, Lee VT. Differential Radial Capillary Action of Ligand Assay (DRaCALA). Curr Protoc Mol Biol. 04 2019;126(1):e84. doi:10.1002/cpmb.84

3. Roelofs KG, Jones CJ, Helman SR, et al. Systematic Identification of Cyclic-di-GMP Binding Proteins in Vibrio cholerae Reveals a Novel Class of Cyclic-di-GMP-Binding ATPases Associated with Type II Secretion Systems. PLoS Pathog. Oct 2015;11(10):e1005232. doi:10.1371/journal.ppat.1005232

4. Wang B, Dai P, Ding D, et al. Affinity-based capture and identification of protein effectors of the growth regulator ppGpp. Nat Chem Biol. 02 2019;15(2):141-150. doi:10.1038/s41589-018-0183-4 5. Mechold U, Potrykus K, Murphy H, Murakami KS, Cashel M. Differential regulation by ppGpp versus pppGpp in Escherichia coli. Nucleic Acids Res. Jul 2013;41(12):6175-89. doi:10.1093/nar/gkt302 6. Wang JD, Sanders GM, Grossman AD. Nutritional control of elongation of DNA replication by (p)ppGpp. Cell. Mar 09 2007;128(5):865-75. doi:10.1016/j.cell.2006.12.043 